\title{
Distinct Neural Substrates of Duration-Based and Beat-Based Auditory Timing
}

\author{
Sundeep Teki, ${ }^{1,2}$ Manon Grube, ${ }^{2}$ Sukhbinder Kumar, ${ }^{1,2}$ and Timothy D. Griffiths ${ }^{1,2}$ \\ ${ }^{1}$ Wellcome Trust Centre for Neuroimaging, University College London, London WC1N 3BG, United Kingdom, and ${ }^{2}$ Newcastle Auditory Group, Medical \\ School, Newcastle University, Newcastle-upon-Tyne NE2 4HH, United Kingdom
}

Research on interval timing strongly implicates the cerebellum and the basal ganglia as part of the timing network of the brain. Here we tested the hypothesis that the brain uses differential timing mechanisms and networks-specifically, that the cerebellum subserves the perception of the absolute duration of time intervals, whereas the basal ganglia mediate perception of time intervals relative to a regular beat. In a functional magnetic resonance imaging experiment, we asked human subjects to judge the difference in duration of two successive time intervals as a function of the preceding context of an irregular sequence of clicks (where the task relies on encoding the absolute duration of time intervals) or a regular sequence of clicks (where the regular beat provides an extra cue for relative timing). We found significant activations in an olivocerebellar network comprising the inferior olive, vermis, and deep cerebellar nuclei including the dentate nucleus during absolute, duration-based timing and a striato-thalamo-cortical network comprising the putamen, caudate nucleus, thalamus, supplementary motor area, premotor cortex, and dorsolateral prefrontal cortex during relative, beat-based timing. Our results support two distinct timing mechanisms and underlying subsystems: first, a network comprising the inferior olive and the cerebellum that acts as a precision clock to mediate absolute, duration-based timing, and second, a distinct network for relative, beatbased timing incorporating a striato-thalamo-cortical network.

\section{Introduction}

The timing network of the brain has been shown to include the cerebellum, basal ganglia, and premotor and prefrontal cortical areas (Lewis and Miall, 2003; Buhusi and Meck, 2005). Among these, the cerebellum and the basal ganglia are consistently activated in perceptual timing tasks (Ivry and Keele, 1989; Harrington et al., 1998). The cerebellum has been specifically implicated in discrete encoding of time intervals (Ivry, 1993; Grube et al., 2010a,b), whereas the basal ganglia have been specifically implicated in the perception of rhythmic sequences with a regular beat (Grahn, 2009).

Along with the cerebellum, the inferior olive is also suggested to be involved in interval timing (Yarom and Cohen, 2002; Xu et al., 2006; Liu et al., 2008). The source of climbing fiber input to the Purkinje cells, the olivary neurons display rhythmic subthreshold membrane potential oscillations (Llinás and Yarom, 1981), which are synchronized by electrical coupling between the olivary cells, resulting in dynamic, functional subgroups that form the basis for a synchronized timing device (Llinas et al., 1974; Yarom and Cohen, 2002). On the other hand, the striatum has anatomical connections with the thalamus and the premotor and prefrontal cortical networks that might allow it to act as a "core timer" within a distinct system for timing (Matell and

Received Oct. 24, 2010; revised Dec. 28, 2010; accepted Jan. 27, 2011.

This work was funded by the Wellcome Trust, UK. We are grateful to the Radiology staff at the Wellcome Trust Centre for Neuroimaging, University College London for their excellent technical support.

Correspondence should be addressed to Sundeep Teki, Wellcome Trust Centre for Neuroimaging, University College London, London WC1N 3BG, UK. E-mail: s.teki@fil.ion.ucl.ac.uk.

DOI:10.1523/JNEUROSCI.5561-10.2011

Copyright $\odot 2011$ the authors $\quad 0270-6474 / 11 / 313805-08 \$ 15.00 / 0$
Meck, 2004; Meck et al., 2008). The precise nature of the timing functions of the olivocerebellar system or the basal ganglia has not been elucidated, and the two systems rarely have been directly compared in the same experiment.

Behavioral work has suggested that timing mechanisms in the brain may depend on the rhythmic context of time intervals (Keele et al., 1989; Monahan and Hirsch, 1990; Yee et al., 1994; Pashler, 2001; McAuley and Jones, 2003). One mechanism we studied is absolute, duration-based timing, where the absolute duration of individual time intervals $\left(\Delta T_{i}\right)$ is encoded discretely like a stopwatch. Our group recently demonstrated that patients with spinocerebellar ataxia type 6 and normal subjects with transcranial magnetic stimulation (TMS) applied over the medial cerebellum were specifically impaired on absolute timing tasks such as comparing the absolute duration of single intervals and not on relative timing tasks based on rhythmic sequences with a regular beat (Grube et al., 2010a,b). We also studied relative, beat-based timing, where the perception of time intervals is facilitated by the presence of a regular beat (Essens and Povel, 1985; Yee et al., 1994) and individual intervals are encoded relative to the beat $\left(\Delta T_{i} / T_{\text {beat }}\right)$. The basal ganglia have consistently been implicated in the perception of time intervals relative to the beat duration (Grahn and Brett, 2007; Grahn and Rowe, 2009).

In this functional magnetic resonance imaging (fMRI) experiment, we asked subjects to compare the duration of the last interval to the penultimate interval and varied the rhythmic context of the preceding intervals to be irregular or regular (Yee et al., 1994; Jones and Yee, 1997; Large and Jones, 1999; Barnes and Jones, 2000) and tested our hypothesis that the olivocerebellar system mediates absolute, duration-based timing, whereas the 
striato-thalamo-cortical system mediates relative, beat-based timing.

\section{Materials and Methods}

Subjects. Eighteen right-handed subjects (aged 18-46 years; mean age, 22.17 years; nine females) with normal hearing and no history of audiological or neurological disorders provided informed written consent and participated in the experiment. Twelve subjects had no formal musical training, whereas six subjects had musical training as a child for an average of 3 years but no current musical training. The study was approved by a National Health Service Research Ethics Committee (ref. 07/Q0905/30).

Stimuli. The stimulus consisted of a sequence of clicks of $0.5 \mathrm{~ms}$ duration and identical loudness (Fig. 1). The number of clicks per sequence was roved between 8 and 11 clicks and the average inter-onset interval (IOI) of sequences was roved between 440 and $560 \mathrm{~ms}$ in steps of $30 \mathrm{~ms}$. The irregular sequence of intervals was created by jittering the IOI by an average of $\pm 15 \%$ and incorporating a time difference of $30 \%$ of the IOI of the penultimate interval, $T_{n-1}$, into the last interval, $T_{n}$. The regular sequence of intervals was characterized by the presence of an isochronous beat with no jitter and had a time difference of $15 \%$ of the IOI between the penultimate (and preceding) and the last interval. Although it is sometimes possible to perceive a beat even in jittered sequences, the above stimulus parameters were carefully tested in pilot experiments to eliminate any beat-based percept in the irregular sequences. Pilot behavioral data suggested that for the same time difference between the final and the penultimate interval, subjects' timing performance on the regular sequences was significantly better than for the irregular sequences. The parameters for the time difference between the last two intervals of the regular and irregular sequences were thus based on extensive pilot testing to ensure comparable performance levels for the timing of the two types of sequences.

Forty irregular and regular sequences each were created that differed according to the total number of intervals in the sequence (four values from 7 to 10 intervals), the number of different interonset intervals (five values: 440, $470,500,530$, and $560 \mathrm{~ms}$ ), and the relative duration of the final interval compared to the penultimate interval (two possibilities: shorter or longer), and the combination of variations in these parameters was completely balanced. Forty silent sequences were also created to be used as control trials to check for auditory cortex activations in this sparse imaging paradigm. All stimuli were created digitally using MATLAB 7.9 software (MathWorks) at a sampling rate of $44.1 \mathrm{kHz}$ and 16 bit resolution.

Task. Subjects were required to judge whether the last interval was shorter or longer than the penultimate interval. Feedback was provided after each trial. The rhythmic context of the intervals before the last two intervals was either irregular or regular (containing a beat) and allowed investigation of the effect of regularity of the rhythmic context on interval timing. For the irregular sequences, we hypothesized that subjects would encode each interval separately, whereas for the regular sequences, we hypothesized that subjects would entrain with the underlying beat and time intervals relative to it.

Experimental design. Before scanning, the subjects read an instruction sheet and had the experimental procedure explained to them. They were not explicitly informed about the difference in the rhythmic context between the two types of sequences. This was done to ensure that subjects performed the timing task without any knowledge of the rhythmic context and to check for corresponding activations in the cerebellum and striatum according to our a priori hypothesis. Subjects were trained on a set of 10-30 practice trials until they achieved a mean accuracy of at least $60 \%$ for both types of sequences.

The task of the subjects was to discriminate between the duration of the last and the second-to-last interval for all the sound sequences. They
A
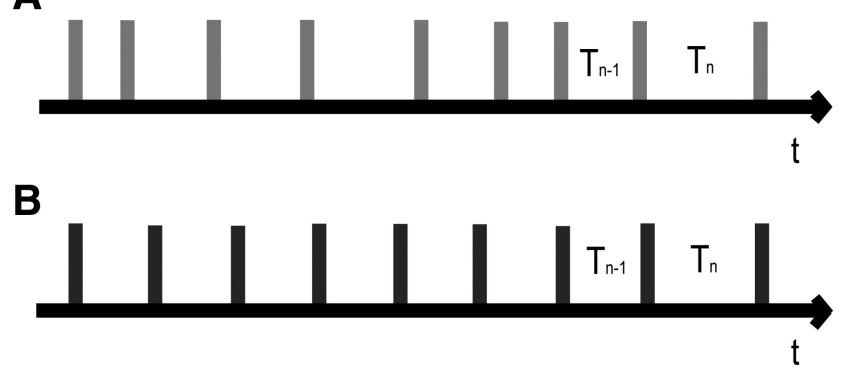

Figure 1. Stimulus and timing task. A, A sequence of clicks with an average of $15 \%$ jitter was used to study absolute, duration-based timing. Subjects were required to compare the duration of the final interval, $T_{n}$, to the penultimate interval, $T_{n-1}$, where the final interval, $T_{n}$, incorporates a difference $(\Delta T)$ of $30 \%$ of the inter-onset interval from that of the preceding interval such that $T_{n}=T_{n-1} \pm \Delta T_{30 \%}$. $B$, A sequence of clicks with no jitter is used to study relative, beat-based timing. Subjects were required to compare the duration of the final interval, $T_{n}$, to the penultimate interval, $T_{n-1}$, where the final interval, $T_{n}$ incorporates a difference $(\Delta T)$ of $15 \%$ of the inter-onset interval from that of the preceding interval such that $T_{n}=T_{n-1} \pm \Delta T_{15 \%}$.

Figure 2. Functional imaging paradigm. The stimulus was presented in quiet using a sparse temporal sampling paradigm. The stimulus occurred 5-8 s after the onset of silence and varied according to the inter-onset interval and the total number of intervals in the sequence. Stimuli were preceded by a variable period of silence on each trial so that the combined duration of silence and $15 \mathrm{~s}$ ) was used to have the peak of the hemodynamic response function optimally time locked to the perceptual timing response $T R=16.44 \mathrm{~s}$

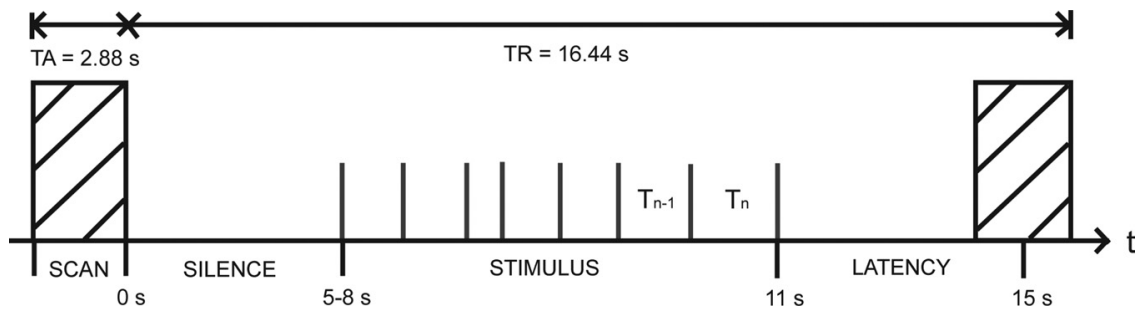

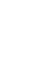


Behavioral data analysis. Subjects' button presses were recorded to obtain a measure of accuracy and reaction time. Responses were registered only if they occurred within the 3 s response window, and only the first button press was evaluated as the response. Both accuracy and reaction times were analyzed separately for the irregular and regular sequences of intervals.

Image acquisition. Gradient-weighted echo planar images (EPIs) were acquired on a 3 Tesla Siemens Allegra MRI scanner using a sparse imaging design with the following parameters: 48 contiguous slices per volume; time to repeat (TR), $16.44 \mathrm{~s}$; time to echo (TE), $30 \mathrm{~ms}$; time for acquisition (TA), $2.88 \mathrm{~s}$; flip angle $\alpha, 90^{\circ}$; matrix size, $64 \times 72$; slice thickness, $2 \mathrm{~mm}$ with $1 \mathrm{~mm}$ gap between slices; and in-plane resolution, $3.0 \times 3.0 \mathrm{~mm}^{2}$. The slices were tilted by $-7^{\circ}$ (transverse $>$ coronal) to obtain full coverage and an optimal signal from the cerebellum. This tilt captured the whole cerebellum in all the subjects tested in the study. Subjects completed one scanning session consisting of 122 volumes. To correct for geometric distortions in the EPI caused by magnetic field variations (Hutton et al., 2002), field maps were acquired for each subject with a double-echo gradient echo field map sequence $\left(\mathrm{TE}_{1}=10.00 \mathrm{~ms}\right.$ and $\mathrm{TE}_{2}=12.46 \mathrm{~ms}$ ). A T1-weighted structural scan was acquired for each subject after the functional scan (Deichmann et al., 2004).

A sparse temporal sampling design was used (Fig. 2) to present the auditory stimuli in quiet and to record responses unaffected by the scanner noise (Belin et al., 1999; Hall et al., 1999). The total duration of the stimulus ranged from 3 to $6 \mathrm{~s}$, depending on the IOI and the total number of intervals in the sequence. Each stimulus was preceded by a variable period of silence so that the combined duration of silence and stimulus was fixed at $11 \mathrm{~s}$ for all trials. Stimulus presentation was followed by a response period of $3 \mathrm{~s}$ and a feedback period of $0.5 \mathrm{~s}$. Volume acquisition started at $t=13.56 \mathrm{~s}$ so that the middle-slice acquisition (with a TA of $2.88 \mathrm{~s}$ ) always occurred at $t=15 \mathrm{~s}$. The time course of the blood oxygenation level-dependent (BOLD) response was tested in pilot experiments and a fixed latency of $4 \mathrm{~s}$ from stimulus offset to the acquisition of the middle slice was selected to arrange for the peak of the slow hemodynamic response function (HRF) to be time locked to the end of the stimulus presentation. This ensured that the peak of the BOLD signal captured the perceptual timing response rather than the subsequent motor response with a minimal overlap in their HRFs.

Image analysis. Imaging data were analyzed using statistical parametric mapping software (SPM8; Wellcome Trust Centre for Neuroimaging). The first two volumes were discarded to control for saturation effects. The resulting 120 volumes were realigned to the first volume and unwarped using the field map parameters. The structural scan was segmented to obtain a bias-corrected structural image, which has more uniform intensities within the different tissue classes: gray matter (GM), white matter (WM), and CSF. The GM, WM, and CSF images were used to skull strip the bias-corrected structural scan, which was coregistered with the mean functional image obtained after realignment. Diffeomorphic Anatomical Registration Through Exponentiated Lie Algebra (DARTEL), an image registration algorithm within SPM8 that provides more precise intersubject alignment and improved fMRI data analysis, was used to create a series of templates using the GM and WM images (Ashburner, 2007). The final template obtained in the previous step was affine registered with the tissue probability maps released with SPM8 so that the resulting spatially normalized images were more closely aligned to Montreal Neurological Institute (MNI) space (Friston et al., 1995a). The resultant images were smoothed with an isotropic Gaussian kernel of $5 \mathrm{~mm}$ full-width at half-maximum.

Statistical analysis was conducted using the general linear model as described by Friston et al. (1995b). The trials based on irregular and regular sequences and the silent trials interleaved within either block were modeled as effects of interest and convolved with an HRF boxcar function. Missed trials were modeled separately as conditions of no interest separately for the irregular and regular sequences and were used to remove unwanted variance. High-pass filtering was not applied because low-frequency variations in the BOLD signal were negligible because of the sparse temporal sampling design.

A whole-brain random-effects model was implemented to account for within-subject variance (Penny and Holmes, 2004). Each individual sub-
Table 1. Stereotactic MNI coordinates for absolute, duration-based timing

\begin{tabular}{|c|c|c|c|c|c|}
\hline Brain area & Hemisphere & $x$ & $y$ & $z$ & $t$-value \\
\hline \multirow[t]{10}{*}{ Inferior olive } & & 0 & -34 & -48 & 4.12 \\
\hline & & 0 & -34 & -51 & 4.11 \\
\hline & & 0 & -34 & -53 & 4.81 \\
\hline & & 0 & -34 & -54 & 5.78 \\
\hline & & 0 & -34 & -57 & 6.27 \\
\hline & & 0 & -36 & -51 & 3.89 \\
\hline & & 0 & -36 & -53 & 4.73 \\
\hline & & 0 & -36 & -54 & 5.77 \\
\hline & & 0 & -36 & -56 & 6.52 \\
\hline & & 0 & -36 & -57 & 6.64 \\
\hline \multirow[t]{4}{*}{ Dentate nucleus } & Right & 9 & -46 & -33 & 6.23 \\
\hline & & 9 & -51 & -30 & 6.06 \\
\hline & Left & -9 & -46 & -33 & 4.87 \\
\hline & & -9 & -51 & -30 & 4.80 \\
\hline \multirow[t]{2}{*}{ Cerebellum } & Right & 24 & -46 & -35 & 7.67 \\
\hline & Left & -11 & -46 & -35 & 5.61 \\
\hline \multirow[t]{2}{*}{ Vermis } & Right & 3 & -45 & -23 & 5.00 \\
\hline & Left & -3 & -45 & -23 & 4.84 \\
\hline \multirow[t]{2}{*}{ Cerebellar lobule IX } & Right & 3 & -51 & -54 & 6.46 \\
\hline & Left & -3 & -51 & -54 & 5.86 \\
\hline \multirow[t]{2}{*}{ Cerebellar lobule X } & Right & 12 & -48 & -39 & 6.28 \\
\hline & Left & -12 & -48 & -39 & 4.80 \\
\hline \multirow[t]{2}{*}{ Superior temporal gyrus } & Right & 36 & 0 & -39 & 7.01 \\
\hline & Left & -32 & 6 & -26 & 5.92 \\
\hline \multirow[t]{2}{*}{ Cochlear nucleus } & Right & 8 & -34 & -48 & 3.98 \\
\hline & Left & -14 & -40 & -37 & 3.92 \\
\hline
\end{tabular}

Local maxima for absolute, duration-based timing are shown at $p<0.001$ (uncorrected).

ject's first-level contrast images were entered into second-level $t$ tests for the primary contrasts of interest: "irregular versus regular" (measure of absolute timing) and regular versus irregular (measure of relative timing). $t$ tests for contrasts of the regular and irregular sequences relative to silent trials were also computed. Functional results are overlaid onto the group-average T1-weighted structural scans, and activations specific to the olivocerebellar system are overlaid on the high-resolution, spatially unbiased infratentorial template (SUIT) atlas of the human cerebellum (Diedrichsen, 2006; Diedrichsen et al., 2009).

\section{Results}

The data were analyzed to test our prediction of a functional dissociation in the neural substrates underlying absolute, duration-based timing and relative, beat-based timing. Behaviorally, we expected no significant differences in performance between the two. For the functional imaging data, we specifically hypothesized the olivocerebellar system to be activated for the timing of the irregular sequences, where the absolute duration of each interval is encoded discretely, and the basal ganglia to be activated for the timing of the regular sequences, where the presence of an isochronous beat provides a beneficial cue for the timing of intervals relative to the beat duration.

\section{Behavioral results}

Behavioral performance for accuracy for the perceptual timing of irregular and regular sequences was measured. The average accuracy for the timing of irregular sequences of intervals was $81.53 \pm$ $12.28 \%$, and the average accuracy for the timing of regular sequences was marginally better at $84.72 \pm 10.64 \%$. The performance scores were not statistically different from one another ( $p>0.05$; two-tailed Student's $t$ test).

The average reaction time for the timing of irregular sequences was $1438 \pm 297 \mathrm{~ms}$, and the average reaction time for the timing of regular sequences was significantly lower at $1275 \pm 312$ ms $(p<0.01$; two-tailed Student's $t$ test $)$. 
A review of the post-scanning subjective reports indicated that the majority of the subjects did not notice any difference in the temporal regularity of the sequences presented to them during the first or the second half of the experiment.

\section{Imaging results}

Absolute, duration-based timing

The analysis for the irregular-versusregular contrast revealed significant bilateral activations in the inferior olive, cerebellar lobules IX and X, vermis, deep cerebellar nuclei including the dentate nucleus, superior temporal gyri, and the cochlear nucleus (see Table 1 ). This pattern of olivocerebellar activations is shown in Figure 3 as overlaid on the SUIT template. Moreover, within the inferior olive, we observed an interesting gradient in activation such that the superior parts of the inferior olive (e.g., $t=6.64$ at coordinates $0,-36,-57)$ were more strongly activated than the inferior areas (e.g., $t=4.15$ at coordinates $0,-36,-47)$.

\section{Relative, beat-based timing}

For the regular-versus-irregular contrast, we found significant bilateral activations in a striato-thalamo-cortical network comprising the putamen, caudate nucleus, thalamus, supplementary motor area (SMA), dorsal premotor cortex, and dorsolateral prefrontal cortex (DLPFC) as shown in Figure 4 (see Table 2). In the striatum, stronger activation was observed in the left putamen and the caudate nucleus.

\section{Dissociation between absolute and} relative timing

The dissociation in the brain areas underlying duration-based timing (above the tentorium) and beat-based timing is revealed by the respective MNI glass brain images at a $t$-value threshold of 4.00 and an extent threshold of 10 voxels (Fig. $5 A, B$ ). The dissociation is highlighted further in Figure $5 C$, where yellow blobs depict olivocerebellar activations for duration-based timing and green blobs depict striatothalamo-cortical activations for beat-based timing on a sagittal section of the mean normalized structural image at $x=7$ $\mathrm{mm}$ and a threshold of $p<0.01$ (uncorrected).

The varied IOI and the number of intervals also had smaller contextual effects on the accuracy and reaction time for durationbased and beat-based timing (supplemental Figs. S1, S2, available at www.jneurosci.org as supplemental material). The highest accuracy was obtained for beat-based timing, with an IOI of $500 \mathrm{~ms}$ $(89.48 \pm 9.86 \%)$, and the lowest reaction times were obtained for beat-based timing, with nine preceding intervals before the last interval ( $1229.70 \pm 385.63 \mathrm{~ms})$.

The contrast images for the regular and irregular trials with respect to the silent trials confirmed activation of auditory cortical and association areas as Heschl's gyrus and planum temporale were ac- tivated bilaterally in both contrasts. This confirmed that the sparse auditory stimuli produced clear auditory cortical activation with no differential activation between conditions.

\section{Discussion}

We used a novel auditory timing task to compare the timing functions of the cerebellum and the basal ganglia. Consistent with our a priori hypothesis, we observed the olivocerebellar and the striato-thalamo-cortical systems to be differentially involved in duration-based and beat-based timing, respectively.

\section{Duration-based versus beat-based timing}

Behavioral work has suggested that duration-based timing and beat-based timing may be functionally different (Pashler, 2001; Ivry et al., 2002; McAuley and Jones, 2003). Pashler (2001) suggested that the brain uses a single interval timer that can be differentially recruited (in a cyclic manner) to generate rhythmic responses. A regular beat can serve as a beneficial cue and aid judgment about temporal changes (Essens and Povel, 


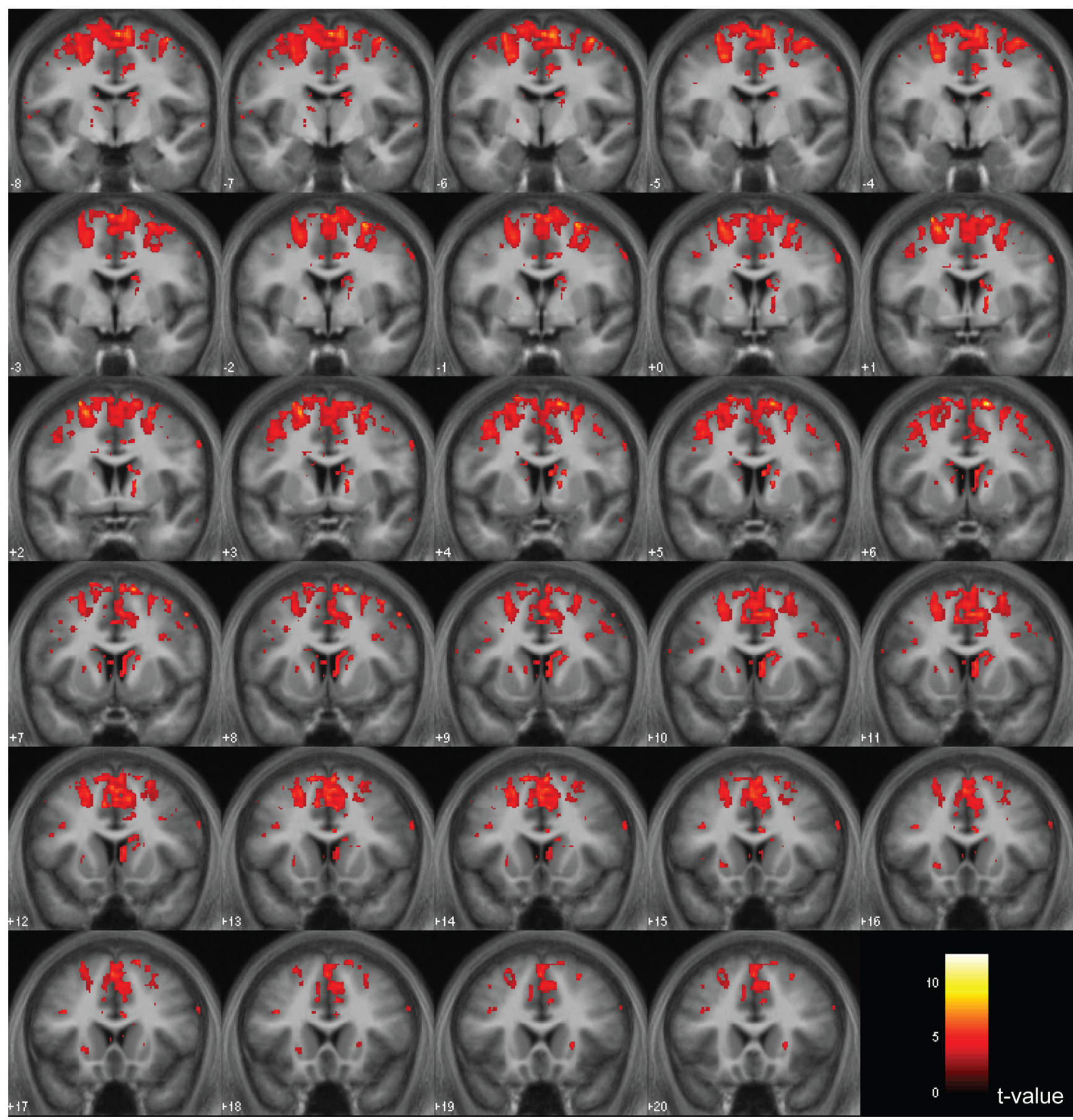

Figure 4. Striatal, thalamic, premotor, and prefrontal activations specific to relative, beat-based timing. BOLD activations for relative, beat-based timing (regular vs irregular) are shown in a series of coronal sections. Significant activations were found in the striatum, thalamus, premotor cortex, SMA, and prefrontal areas including the DLPFC at a threshold of $p<0.001$ (uncorrected). The sections show activations at every $1 \mathrm{~mm}$ from $y=-8 \mathrm{~mm}$ to $=+20 \mathrm{~mm}$ as overlaid on the average normalized structural scan of all subjects. The strength of activations ( $t$-value) is graded according to the color scheme at the bottom right.

1985; Yee et al., 1994; Grahn and Brett, 2007). We also found that subjects performed better for regular than for irregular sequences when presented with identical time difference between the last two intervals (see Materials and Methods).

The existence of duration-based and beat-based timing mechanisms is supported by previous work. Monahan and Hirsch (1990) suggested a similar difference in measures of "absolute" ( $\Delta t$; fixed absolute difference) and "relative" ( $\Delta t / t$; constant proportion) discrimination of temporal changes in a sequence of intervals. Yee et al. (1994) showed that contextual factors influ- ence listeners' performance on a time-change detection task, in which they were more accurate in detecting temporal changes in regular sequences than in irregular sequences. Here, we induced a preceding rhythmic context (Large and Jones, 1999; Barnes and Jones, 2000) and varied it to be either irregular or regular to isolate duration-based and beat-based timing mechanisms, respectively.

In relation to the timing of movements, Ivry et al. (2002) described "event timing" as being associated with a discrete representation of time intervals that is needed in perceptual timing 
Table 2. Stereotactic MNI coordinates for relative, beat-based timing

\begin{tabular}{llrrrr}
\hline Brain area & Hemisphere & \multicolumn{1}{c}{$x$} & $y$ & $z$ & $t$-value \\
\hline Caudate nucleus & Right & 14 & 9 & 18 & 5.69 \\
\multirow{3}{*}{ Putamen } & Left & -14 & 6 & 21 & 4.32 \\
& Right & 23 & 21 & 0 & 4.28 \\
Internal capsule & Left & -24 & 15 & -2 & 4.55 \\
Thalamus & Right & 15 & 3 & 6 & 6.99 \\
& Left & -15 & 3 & 12 & 3.16 \\
Pre-SMA/SMA & Right & 9 & -15 & 12 & 6.44 \\
& Left & -9 & -21 & 12 & 6.06 \\
Premotor cortex & Right & 15 & 6 & 64 & 12.61 \\
\multirow{3}{*}{ Dorsolateral prefrontal cortex } & Left & -2 & -3 & 67 & 6.61 \\
\multirow{3}{*}{ Superior temporal gyrus } & Right & 38 & -6 & 60 & 8.04 \\
& Left & -41 & 5 & 51 & 5.53 \\
& Right & 35 & 9 & 31 & 4.62 \\
& Left & -38 & 18 & 25 & 5.30 \\
& Right & 66 & -39 & 3 & 5.82 \\
& Left & -41 & -30 & 9 & 6.72 \\
\hline
\end{tabular}

Local maxima for relative, beat-based timing are shown at $p<0.001$ (uncorrected).

tasks such as duration discrimination and "emergent timing" as being associated with the processing of a temporal regularity or a beat in the sequence to be timed. The authors suggested that tasks requiring an explicit or discrete representation of time intervals such as the temporal control of a series of discrete movements involve the cerebellum and that temporal control of rhythmic and continuous movements might be subserved by cortical networks and not the cerebellum (Spencer et al., 2003). Complementary to this dissociation in timing of movements, here we report a functional dissociation in perceptual timing with no explicit linked motor output such as tapping.

\section{Role of the olivocerebellar system}

The inferior olive has remarkable cellular and network properties that make it an ideal generator of temporal patterns (Yarom and Cohen, 2002; Jacobson et al., 2008). The intrinsic voltage-gated conductances present in olivary neurons sustain rhythmic subthreshold membrane potential oscillations at $\sim 5-15 \mathrm{~Hz}$ and enable them to generate a timing signal (Llinás and Yarom, 1981). These oscillations are synchronized by electrical coupling between the olivary cells and organize them into temporally coherent groups (Llinas et al., 1974). The electrical coupling coefficient is controlled by GABAergic projections from the deep cerebellar nuclei, which synapse directly on the gap junctions between olivary cells and organize them into dynamic, functional subgroups. The deep cerebellar nuclei receive inhibitory input from the cerebellar Purkinje cells, which in turn receive climbing fiber afferents from the olivary cells, thus forming a dynamic network capable of producing accurate timing signals (Welsh et al., 1995).

Inferior olive activity has been reported previously in fMRI studies of perceptual timing based on visual sequences (Xu et al., 2006; Liu et al., 2008). Here, we observed significant inferior olive activation for auditory timing of irregular sequences without an apparent beat in contrast to the timing of regular sequences with an isochronous beat. Xu et al. (2006) found similar inferior olive activity in a perceptual timing task when they contrasted timing of metrical rhythmic sequences to timing of isochronous sequences. Moreover, the inferior olive has been suggested to mediate adaptive timing of the classically conditioned, eye-blink response and the vestibulo-ocular reflex (Ito et al., 1970). The sole source of climbing fiber input to the Purkinje cells, the inferior olive has been shown to subserve error-based learning by
A Activations for absolute, duration-based timing
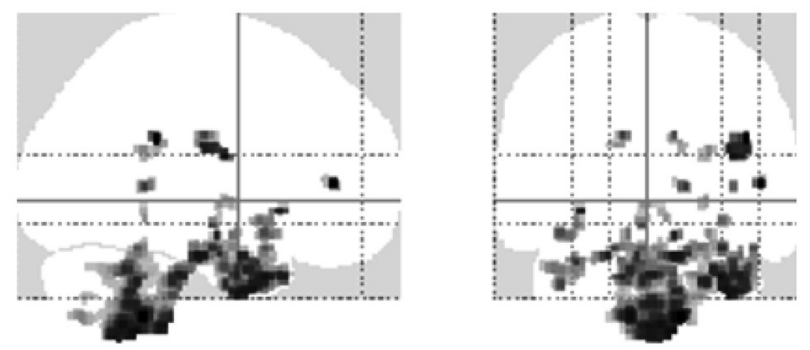

B Activations for relative, beat-based timing
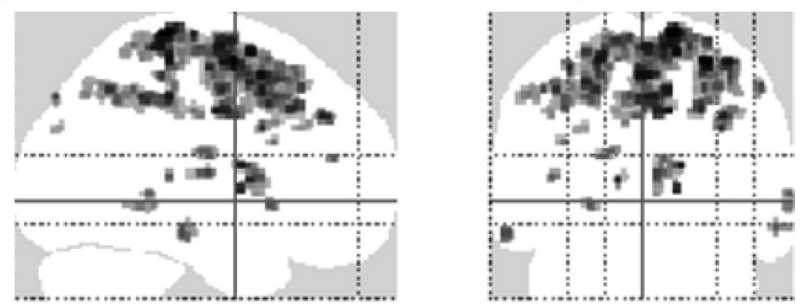

C Dissociation between absolute and relative timing

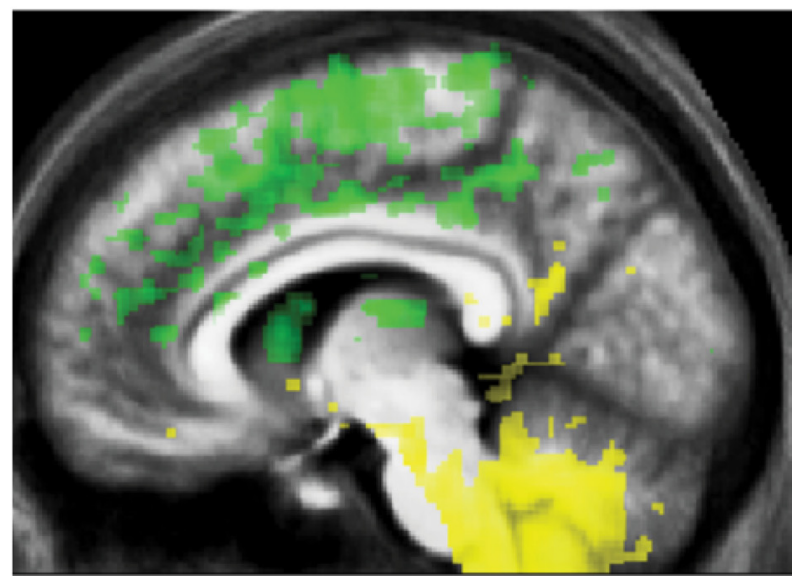

Figure 5. Dissociation between neural substrates underlying absolute and relative timing. $A$, Glass brain image in MNI space showing activations for absolute, duration-based timing (irregular vs regular) at a $t$-value threshold of 4.00 and an extent threshold of 10 voxels. B, Glass brain image in MNI space showing activations for relative, beat-based timing (regular vs irregular) at a $t$-value threshold of 4.00 and an extent threshold of 10 voxels. C, Activations for absolute timing are depicted in yellow, and activations for relative timing are shown in green on a sagittal section of the average normalized structural image at $x=+7 \mathrm{~mm}$ and a threshold of $p<0.01$ (uncorrected) to show the clear differences in the brain bases for absolute and relative timing, respectively.

mediating long-term depression of the parallel fiber input to the Purkinje cells (Ito et al., 1982). Together, these results suggest a possible role for the olivocerebellar system in detecting errors in the regular operation of a beat-based timer in the striatum.

In addition to the inferior olive, we found strong activations in the cerebellum, which has been widely implicated in interval timing (Ivry and Keele, 1989; Nichelli et al., 1996; Ivry et al., 2002; Grube et al., 2010a,b). Its specific role in absolute, duration-based timing was demonstrated in patients with spinocerebellar ataxia type 6 by Grube et al. (2010a), who used a battery of auditory timing tasks based on duration-based or beat-based timing mechanisms and found a significant impairment only on the duration-based timing tasks (e.g., comparing the absolute duration of single intervals) and not on relative timing of rhythmic sequences based on a regular beat. 
This pattern of results was replicated in a TMS study on normal subjects who were selectively impaired on the same absolute timing task after application of TMS over the medial cerebellum (Grube et al., 2010b).

We also found activations in the dentate nucleus, the main output nucleus of the lateral cerebellar hemispheres, which has been postulated to mediate perceptual timing and sensory discrimination (Ivry and Keele, 1989; Gao et al., 1996; Middleton and Strick, 2001; Gooch et al., 2010). It can also influence timing functions in the basal ganglia through its disynaptic projections to an input stage of the basal ganglia, the striatum (Hoshi et al., 2005). The observed activation in the dentate nucleus is also significant in light of its projections to the principal olive (Kalil, 1979), which is suggested to be preferentially involved in perceptual timing, unlike the dorsal and medial accessory olives, which are instead suggested to mediate motor timing (Xu et al., 2006).

The inferior olive receives visual input (Takeda and Maekawa, 1980; Leonard et al., 1988), auditory input from the cochlear nucleus (Kandler and Herbert, 1991), and bilateral afferents from the caudate nucleus and the cerebral cortex (Walberg, 1956). Together, these results suggest that the inferior olive in association with the cerebellum is in a suitable position to serve as a supramodal, duration-based timer.

\section{Role of the striato-thalamo-cortical system}

The striatum and the pre-SMA/SMA, which are strongly connected through striato-thalamo-cortical loops, have been implicated in many studies of beat perception with a specific role for the putamen and caudate nucleus in beat perception (Grahn and Brett, 2007; Grahn, 2009). We observed significant activations in the striatum, thalamus, the SMA, and the DLPFC, suggesting a role for the core, striato-thalamo-cortical timing network in beat-based timing (Macar et al., 2004; Meck et al., 2008). Our behavioral results of higher accuracy and speed for beat-based timing (optimal performance at a beat rate of $2 \mathrm{~Hz}$ ) (see supplemental Figs. S1 A, S2 A, available at www.jneurosci.org as supplemental material) suggest that a beat-based clock is more efficient.

The role of striatum is substantiated by neuropharmacological evidence suggesting that the nigrostriatal dopaminergic system is critical for interval timing. Dopaminergic manipulations are known to affect timing functions (Meck, 1996) and it has been shown that 6-hydroxydopamine lesions in the substantia nigra pars compacta or in the caudate/putamen eliminated timing functions (Meck, 2006). Additionally, the role of dopamine in timing is highlighted by impaired performance of patients of Parkinson's disease on time (Artieda et al., 1992) and rhythmic discrimination tasks (Grahn and Brett, 2009).

Furthermore, the basal ganglia have been suggested to monitor activity in the striato-thalamo-cortical circuits and perform coincidence detection of signal-specific patterns of activity in working memory (Buhusi and Meck, 2005). The coincidental activation of medium spiny neurons in the basal ganglia by cortical oscillators has led to the suggestion of timing as an emergent property of the striato-thalamo-cortical network (Matell and Meck, 2004).

The SMA has been implicated widely in time processing (Macar et al., 2002, 2004, 2006) as well as beat processing (Grahn and Brett, 2007; Chen et al., 2008; Grahn and McAuley, 2009) and plays a key role in time perception as part of the striato-thalamocortical pathway. The SMA is one of the primary projection sites of the striatum to the cortex via the globus pallidus and the thalamus, and its suggested role in perceptual timing is further supported by its direct connections with the putamen and caudate nucleus (Jürgens, 1984) and the dentate nucleus of the cerebellum (Akkal et al., 2007).

We also observed significant activation of the DLPFC, specifically for the timing of the regular sequences containing a beat. The DLPFC receives inputs from both the dentate nucleus of the cerebellum (Middleton and Strick, 2001) and the basal ganglia (Middleton and Strick, 2002). Its activation is consistent with its proposed timing role as part of the striato-thalamo-cortical network (Buhusi and Meck, 2005), where it may facilitate beat-based timing by encoding the beat duration into working memory.

\section{Conclusions}

We found the timing network of the brain to be dissociated based on the rhythmic context of time intervals, such that the olivocerebellar system mediates absolute, duration-based timing while the striato-thalamo-cortical system subserves relative, beat-based timing.

\section{References}

Akkal D, Dum RP, Strick PL (2007) Supplementary motor area and presupplementary motor area: targets of basal ganglia and cerebellar output. J Neurosci 27:10659-10673.

Artieda J, Pastor MA, Lacruz F, Obeso JA (1992) Temporal discrimination is abnormal in Parkinson's disease. Brain 115:199-210.

Ashburner J (2007) A fast diffeomorphic image registration algorithm. Neuroimage 38:95-113.

Barnes R, Jones MR (2000) Expectancy, attention, and time. Cogn Psychol 41:254-311.

Belin P, Zatorre RJ, Hoge R, Evans AC, Pike B (1999) Event-related fMRI of the auditory cortex. Neuroimage 10:417-429.

Buhusi CV, Meck WH (2005) What makes us tick? Functional and neural mechanisms of interval timing. Nat Rev Neurosci 6:755-765.

Chen JL, Penhune VB, Zatorre RJ (2008) Listening to musical rhythms recruits motor regions of the brain. Cereb Cortex 18:2844-2854.

Deichmann R, Schwarzbauer C, Turner R (2004) Optimisation of the 3D MDEFT sequence for anatomical brain imaging: technical implications at 1.5 and $3 \mathrm{~T}$. Neuroimage 21:757-767.

Diedrichsen J (2006) A spatially unbiased atlas template of the human cerebellum. Neuroimage 33:127-138.

Diedrichsen J, Balsters JH, Flavell J, Cussans E, Ramnani N (2009) A probabilistic MR atlas of the human cerebellum. Neuroimage 46:39-46.

Essens PJ, Povel DJ (1985) Metrical and nonmetrical representations of temporal patterns. Percept Psychophys 37:1-7.

Friston KJ, Ashburner J, Frith CD, Poline J, Heather JD, Frackowiak RSJ (1995a) Spatial registration and normalization of images. Hum Brain Mapp 3:165-189.

Friston KJ, Holmes AP, Worsley KJ, Poline J, Frith CD, Frackowiak RSJ (1995b) Statistical parametric maps in functional imaging: a general linear approach. Hum Brain Mapp 2:189-210.

Gao JH, Parsons LM, Bower JM, Xiong J, Li J, Fox PT (1996) Cerebellum implicated in sensory acquisition and discrimination rather than motor control. Science 272:545-547.

Gooch CM, Wiener M, Wencil EB, Coslett HB (2010) Interval timing disruptions in subjects with cerebellar lesions. Neuropsychologia 48:1022-1031.

Grahn JA (2009) The role of the basal ganglia in beat perception: neuroimaging and neuropsychological investigations. Ann N Y Acad Sci 1169:35-45.

Grahn JA, Brett M (2007) Rhythm and beat perception in motor areas of the brain. J Cogn Neurosci 19:893-906.

Grahn JA, Brett M (2009) Impairment of beat-based rhythm discrimination in Parkinson's disease. Cortex 45:54-61.

Grahn JA, McAuley JD (2009) Neural bases of individual differences in beat perception. Neuroimage 47:1894-1903.

Grahn JA, Rowe JB (2009) Feeling the beat: premotor and striatal interactions in musicians and nonmusicians during beat perception. J Neurosci 29:7540-7548.

Grube M, Cooper FE, Chinnery PF, Griffiths TD (2010a) Dissociation of duration-based and beat-based auditory timing in cerebellar degeneration. Proc Natl Acad Sci U S A 107:11597-11601.

Grube M, Lee K-H, Griffiths TD, Barker AT, Woodruff PW (2010b) Trans- 
cranial magnetic theta-burst stimulation of the human cerebellum distinguishes absolute, duration-based timing from relative, beat-based perception of subsecond time intervals. Front Psychology 1:171.

Hall DA, Haggard MP, Akeroyd MA, Palmer AR, Summerfield AQ, Elliott MR, Gurney EM, Bowtell RW (1999) "Sparse" temporal sampling in auditory fMRI. Hum Brain Mapp 7:213-223.

Harrington DL, Haaland KY, Hermanowicz N (1998) Temporal processing in the basal ganglia. Neuropsychology 12:3-12.

Hoshi E, Tremblay L, Féger J, Carras PL, Strick PL (2005) The cerebellum communicates with the basal ganglia. Nat Neurosci 8:1491-1493.

Hutton C, Bork A, Josephs O, Deichmann R, Ashburner J, Turner R (2002) Image distortion correction in fMRI: a quantitative evaluation. Neuroimage 16:217-240.

Ito M, Highstein SM, Fukuda J (1970) Cerebellar inhibition of the vestibulo-ocular reflex in rabbit and cat and its blockage by picrotoxin. Brain Res 17:524-526.

Ito M, Sakurai M, Tongroach P (1982) Climbing fibre induced depression of both mossy fibre responsiveness and glutamate sensitivity of cerebellar Purkinje cells. J Physiol 324:113-134.

Ivry R (1993) Cerebellar involvement in the explicit representation of temporal information. Ann N Y Acad Sci 682:214-230.

Ivry RB, Keele SW (1989) Timing functions of the cerebellum. J Cogn Neurosci 1:136-152.

Ivry RB, Spencer RM, Zelaznik HN, Diedrichsen J (2002) The cerebellum and event timing. Ann N Y Acad Sci 978:302-317.

Jacobson GA, Rokni D, Yarom Y (2008) A model of the olivo-cerebellar system as a temporal pattern generator. Trends Neurosci 31:617-625.

Jones MR, Yee W (1997) Sensitivity to time change: the role of context and skill. J Exp Psychol Hum Percept Perform 23:693-709.

Jürgens U (1984) The efferent and afferent connections of the supplementary motor area. Brain Res 300:63-81.

Kalil K (1979) Projections of the cerebellar and dorsal column nuclei upon the inferior olive in the rhesus monkey: an autoradiographic study. J Comp Neurol 188:43-62.

Kandler K, Herbert H (1991) Auditory projections from the cochlear nucleus to pontine and mesencephalic reticular nuclei in the rat. Brain Res 562:230-242.

Keele SW, Nicoletti R, Ivry RI, Pokorny RA (1989) Mechanisms of perceptual timing: beat-based or interval-based judgments? Psychol Res 50:251-256.

Large EW, Jones MR (1999) The dynamics of attending: how we track time varying events. Psychol Rev 106:119-159.

Leonard CS, Simpson JI, Graf W (1988) Spatial organization of visual messages of the rabbit's cerebellar flocculus. I. Typology of inferior olive neurons of the dorsal cap of Kooy. J Neurophysiol 60:2073-2090.

Lewis PA, Miall RC (2003) Distinct systems for automatic and cognitively controlled time measurement: evidence from neuroimaging. Curr Opin Neurobiol 13:250-255.

Liu T, Xu D, Ashe J, Bushara K (2008) Specificity of inferior olive response to stimulus timing. J Neurophysiol 100:1557-1561.

Llinás R, Yarom Y (1981) Electrophysiology of mammalian inferior olivary neurones in vitro. Different types of voltage-dependent ionic conductances. J Physiol 315:549-567.

Llinas R, Baker R, Sotelo C (1974) Electrotonic coupling between neurons in cat inferior olive. J Neurophysiol 37:560-571.
Macar F, Lejeune H, Bonnet M, Ferrara A, Pouthas V, Vidal F, Maquet P (2002) Activation of the supplementary motor area and of attentional networks during temporal processing. Exp Brain Res 142:475-485.

Macar F, Anton JL, Bonnet M, Vidal F (2004) Timing functions of the supplementary motor area: an event-related fMRI study. Brain Res Cogn Brain Res 21:206-215.

Macar F, Coull J, Vidal F (2006) The supplementary motor area in motor and perceptual time processing: fMRI studies. Cogn Process 7:89-94.

Matell MS, Meck WH (2004) Cortico-striatal circuits and interval timing: coincidence detection of oscillatory processes. Brain Res Cogn Brain Res 21:139-170.

McAuley JD, Jones MR (2003) Modeling effects of rhythmic context on perceived duration: a comparison of interval and entrainment approaches to short-interval timing. J Exp Psychol Hum Percept Perform 29:1102-1125.

Meck WH (1996) Neuropharmacology of timing and time perception. Brain Res Cogn Brain Res 3:227-242.

Meck WH (2006) Neuroanatomical localization of an internal clock: a functional link between mesolimbic, nigrostriatal, and mesocortical dopaminergic systems. Brain Res 1109:93-107.

Meck WH, Penney TB, Pouthas V (2008) Cortico-striatal representation of time in animals and humans. Curr Opin Neurobiol 18:145-152.

Middleton FA, Strick PL (2001) Cerebellar projections to the prefrontal cortex of the primate. J Neurosci 21:700-712.

Middleton FA, Strick PL (2002) Basal-ganglia 'projections' to the prefrontal cortex of the primate. Cereb Cortex 12:926-935.

Monahan CB, Hirsh IJ (1990) Studies in auditory timing: 2. Rhythm patterns. Percept Psychophys 47:227-242.

Nichelli P, Alway D, Grafman J (1996) Perceptual timing in cerebellar degeneration. Neuropsychologia 34:863-871.

Pashler H (2001) Perception and production of brief durations: beat-based versus interval-based timing. J Exp Psychol Hum Percept Perform 27:485-493.

Penny W, Holmes AP (2004) Random-effects analysis. In: Human brain function (Frackowiak RS, Friston KJ, Frith CD, Dolan RJ, Price CJ, eds), pp 843-850. San Diego: Academic.

Spencer RM, Zelaznik HN, Diedrichsen J, Ivry RB (2003) Disrupted timing of discontinuous but not continuous movements by cerebellar lesions. Science 300:1437-1439.

Takeda T, Maekawa K (1980) Bilateral visual inputs to the dorsal cap of inferior olive: differential localization and inhibitory interactions. Exp Brain Res 39:461-471.

Walberg F (1956) Descending connections to the inferior olive; an experimental study in the cat. J Comp Neurol 104:77-173.

Welsh JP, Lang EJ, Suglhara I, Llinás R (1995) Dynamic organization of motor control within the olivocerebellar system. Nature 374:453-457.

Xu D, Liu T, Ashe J, Bushara KO (2006) Role of the olivo-cerebellar system in timing. J Neurosci 26:5990-5995.

Yarom Y, Cohen D (2002) The olivocerebellar system as a generator of temporal patterns. Ann N Y Acad Sci 978:122-134.

Yee W, Holleran S, Jones MR (1994) Sensitivity to event timing in regular and irregular sequences: influences of musical skill. Percept Psychophys 56:461-471. 